\title{
Keystrokes and Clicks: Measuring Stress on E-learning Students
}

\author{
Manuel Rodrigues ${ }^{1,2}$, Sérgio Gonçalves ${ }^{3}$, Davide Carneiro ${ }^{3}$, Paulo Novais ${ }^{3}$, \\ Florentino Fdez-Riverola ${ }^{2}$ \\ ${ }^{1}$ Informatics Group, Secondary School Martins Sarmento, Guimarães, Portugal \\ ${ }^{2}$ Informatics Department, University of Vigo, Ourense, Spain \\ ${ }^{3}$ Informatics Department/ Computer Science and Technology Center, University \\ of Minho, Braga, Portugal
}

\begin{abstract}
In traditional learning, teachers can easily get an insight into how their students work and learn and how they interact in the classroom. However, in online learning, it is more difficult for teachers to see how individual students behave. With the enormous growing of e-learning platforms, as complementary or even primary tool to support learning in organizations, monitoring students' success factors becomes a crucial issue. In this paper we focus on the importance of stress in the learning process. Stress detection in an E-learning environment is an important and crucial factor to success. Estimating, in a non-invasive way, the students' levels of stress, and taking measures to deal with it, is then the goal of this paper. Moodle, by being one of the most used e-learning platforms is used to test the log tool referred in this work.
\end{abstract}

Keywords: E-learning, Behavioral Analysis, Stress, Moodle

\section{Introduction}

When a student attends an electronic course, the interaction between student and teacher, without all its non-verbal interactions, is poorer. Thus the assessment of feelings and attitudes by the teacher becomes more difficult. In that sense, the use of technological tools for teaching, with the consequent teacher-student and student-student separation, may represent a risk as a significant amount of context information is lost. Since students' effectiveness and success in learning is highly related to their mood while doing it, such issues should be taken into account when in an E-learning environment. In a traditional classroom, the teacher can detect and even forecast that some negative situation is about to occur and take measures accordingly to mitigate such situation. When in a virtual environment, such actions are impossible.

Stress, in particular, can play an important (usually negative) role in education 
[1-2]. In that sense, its analysis in an E-learning environment assumes greater importance. Using physiological sensors could be a solution for stress detection. However, the use of visible and invasive sensors induces itself a certain degree of stress. In this work, we extract information from keyboard strokes and mouse movement to generate important information about students' mood towards learning. We are developing a modular tool, able to estimate the level of stress of human users in a non-intrusive way. Our goal is to develop a dynamic stress estimation model that, while making use of this context information, will allow teachers to adapt strategies in search for increased success in learning.

\section{Dynamic Student Assessment Module}

As stated, stress has a significant influence in E-learning performance. To mitigate such problems, several research studies have been carried out. In [3,4] frameworks are proposed where the goal is to obtain an external module to be linked to the Moodle platform, enabling the detection of student's affective states and learning styles in order to really know each student and present contents accordingly. A similar affective module will be responsible for gathering all this information, and derive students' mood (e.g. states of mind or emotion, a particular inclination or disposition to learn something) in order to present relevant clues for a personalization and recommendation module, to be developed in future work. Figure 1 depicts the Dynamic Student Assessment Module (DSAM). Not detailed in this work is the Personalization and Recommendation Module that will be subject of future work. Furthermore, particular attention will be given to stress detection through keyboard and mouse.

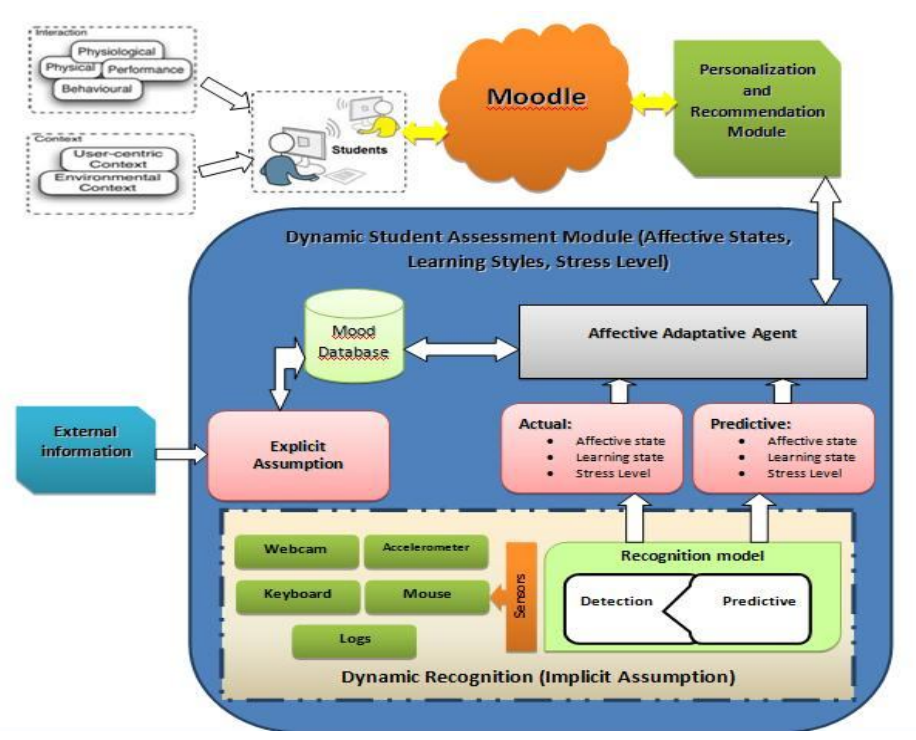

Fig. 1. Dynamic Student Assessment Module 
The Dynamic Student Assessment Module has two sub-modules: explicit assumption and Dynamic Recognition (implicit assumption), whose function is to detect student's mood, maintaining that information (actual and past) in the mood database. This information will be used by another sub-module, the affective adaptive agent, to provide relevant information to the platform and to the mentioned personalization module. This allows actual students' mood information to be displayed in the Moodle platform, and to be used to personalize instruction according to the specific student, enabling Moodle to act differently with different students, and also to act differently to the same student, according to his/her past and present mood. Here, we refer to mood as the actual "willing" of the student to learn, which incorporates his/her affective state, learning style and level of stress.

Each student interacts with Moodle from his/her own real environment, when attending a course. This environment is equipped with sensors and devices that acquire different kind of information from the student in a non-intrusive way. While the student conscientiously interacts with the system and takes his/her decisions and actions, a parallel and transparent process takes place in which this information is used by the Dynamic Student Assessment Module. This module, upon converting the sensory information into useful data, allows for a contextualized analysis of the operational data of the students. This contextualized analysis is performed by the Dynamic Student Assessment Module. Then, the student's profile is updated with new data, and the teacher responsible for that course receives feedback from this module.

To accomplish this, various research works of the research team are being integrated, touching areas such as facial recognition, behavioral analysis and noninvasive stress assessment. Two newly developed modules are explained next, with particular emphasis on mouse and keyboard logs.

\subsection{Explicit and Implicit Mood Assumption}

One of the easiest ways of knowing a student's mood is by making explicit questions. Surprisingly, this may not be the most accurate way: not always the answers obtained reveal the accurate state of the student. However, we can still use questionnaires as a way of gathering some useful information. An explicit mood assumption agent could periodically pose some questions, preferably in a visual way, for the student to upgrade his/her mood to the system. This configures the Explicit Mood Assumption module. Several other research works have been carried out to detect student's mood explicitly [5].

A more interesting approach would be to infer such information. This is done under the Implicit Mood Assumption module. The aim of this sub-module is to monitor the interactions between the student and the system in order to infer the students' mood without being intrusive, that is, without the student being aware of the analysis being performed. Four key aspects are considered, although only two are in the scope of this this work: facial analysis, mouse analysis, keyboard analysis, and $\log$ analysis. As web cams tend to be standard equipment in 
computers nowadays, the goal is to use them to help infer emotions from the user. Mouse movements can also help predict the state of mind of the user, as well as keyboard usage patterns. Finally, analyzing the past interactions of the student through the logs of Moodle makes it possible to infer some of the information we are looking for.

The way a user types may indicate his/her state of mind and level of stress. Pressing keys hard and rapidly could indicate an altered state such as anger, while taking too much time may mean sadness. The same occurs with mouse movements. A similar system that monitors users' behavior from standard input devices, like the keyboard or the mouse, is proposed by [6]. The features analyzed include: the number of mouse clicks per minute, the average duration of mouse clicks (from the button-down to the button-up event), the maximum, minimum and average mouse speeds, the keystroke rate (strokes per second), the average duration of a keystroke (from the key-down to the key-up event) and performance measurements. [7] includes keyboard stroke information in order to improve the accuracy of visual-facial emotion recognition.

The level of stress of the students assumes greater importance due to its correlation to success. The focus of this work is thus on devices capable of acquiring data related to stress. The following sources of information (from now on designated sensors) acquired from the respective devices are:

- Click accuracy - a comparison between clicks in active controls versus clicks in passive areas (e.g. without controls, empty areas) in which there is no sense in clicking. This information is acquired from the mouse.

- Click duration - this represents the time span between the beginning and the end of the click event. This data is acquired from the mouse.

- Amount of movement - the amount of movement represents how and how much the student is moving inside the environment. An estimation of the amount of movement from the video camera is built. The image processing stack uses the principles established by [8] and uses image difference techniques to calculate the amount of movement between two consecutive frames [9] .

- Mouse movement - the amount of mouse movement represents the pattern in which the student moves the mouse (e.g. low amplitude quick movements of the mouse may indicate a high level of stress). This data are acquired from the mouse.

- Mouse clicks - the amount of mouse clicks and its frequency is useful for building an estimation of how much the student is moving around the screen and where he/she clicks. This data is acquired from the mouse.

- Keyboard strokes - frequency and intensity of the use of the keyboard. Frequent backspaces may indicate errors, high keyboard stroke may suggest experienced user (student) as opposed to low keyboard strokes. Stroke intensity (if keyboards allow it) may also be considered. This data is acquired from the keyboard.

Using an E-learning platform requires computers and peripherals for 
interaction. The most common peripherals nowadays are the keyboard and the mouse. Commonly, these interaction instruments are present in classrooms that are used to work with E-learning platforms and also in our homes and offices. Hence the increased advantage of their use for estimating stress.

Concerning the keyboard, there are currently many consolidated studies that point out the accuracy of keystroke analysis, allowing even users' recognition [10]. An area known as keystrokes dynamics aims at the recognition of users through the password provided as well as the correct rhythm of character input [11]. Thus, the changing of keystroke rhythm is a crucial factor to be considered in stress analysis [12].

The cases presented demonstrate how well the process of using keystroke log software packages is regimented. In a normal environment it is possible, using this type of approach in a transparent way, to collect data for later analysis. The user does not feel any intrusion into their actions using the e-learning environment.

The second peripheral that is used very often is the mouse. Its use is already taken for granted in interaction with learning platforms. Hence, the data analysis resulting from the use of this device is of utmost importance. There are already documented experiences of using mouse tracking software in areas of psychological analysis [13]. The coordinates (log coordinates of user interaction), the frequency of movements (log each time interaction) shows the state of the user, whether it will be stressed or normal.

Another area in which mouse tracking is fundamental and a key factor in decision-making is web browsing [14]. It is possible to log the cursor path that users create while browsing web pages. Presently it is also possible to measure the pressure exerted in a device such as a mouse [15]. This gives the possibility to detect actions of different pressure, of users in different psychological states. Mouse tracking should thus be, along with the use of the keyboard, one of the main points of data analysis for the determination of stress. It becomes clear that the possibilities for data analysis are feasible and reliable.

At this point one of the major difficulties and failures that Moodle presents is its $\log$ tool that complicates the analysis of actions and the knowledge of when that action took place. It is not possible to get, with the required certainty, the analysis of users' activity with the main focus on the type of interface used and the type of movement performed with this tool. In addition to this factor, there is the absence of date/time registration, so that the frequency of activity analysis could be determined. Thus, a log tool was developed in C\# language. This is a simple but powerful application which enables to acquire data of users' actions and register them in a log file.

The record of the actions taken with the keyboard follows the logic of recording the type of action with key (Key Up or Key Down) and adding to the information the moment of key usage, in milliseconds.

Concerning the mouse, the registration of actions considers the movement, the clicks, the scrolls and, of course, the time which each of these events took place and their coordinates. This application, that is running in background, creates a log describing the interaction of the student with the Moodle platform. 


\section{Data analysis}

We used ten programming students, as our test group, in order to validate the possibility of detecting users' stress through the analysis of mouse and keyboard usage. The log tool mentioned earlier was used to assess the activity of this group when using a computer. The tested students were not aware of the existence of such tool running on the background of their computers. First, an assignment was given, with no time restrictions and with no influence in terms of difficulty to the student. The correspondent data was collected and analysed. Secondly, at a later moment, the same group was given an assignment, very similar to the first one, but with many constraints introduced. There was a time limit, they were told that the resulting work would have a major importance on their classification, and this group was intentionally submitted to stressing and upsetting factors. The results obtained are clarified in the following figures and table.

This allowed to derive knowledge from keyboard and mouse data. Concerning mouse movements, we found that in the first situation (where a student accomplished a task with no restrictions whatsoever and with no concerns of grade) there were considerably less mouse movements and keyboard usage than when the student had the mentioned restrictions (in terms of work volume, difficulty, influence on final grade), as stated in Table 1. A more detailed analysis of the gathered information while prosecuting the proposed activities allows the evaluation of the kind of usage of the two devices (mouse and keyboard).

Tab. 1. Type of movements during tasks.

\begin{tabular}{|ccc|}
\hline Type of movement & Stress less & Stressed \\
\hline KD & 9 & 30 \\
\hline KU & 9 & 30 \\
\hline MD & 31 & 50 \\
\hline MOV & 2493 & 5427 \\
\hline MU & 31 & 50 \\
\hline MW & 43 & 208 \\
\hline
\end{tabular}

KD: key down; KU: key up; MD: mouse down; UM: mouse up; MW: mouse wheel ; Mov: mouse movement

It is concluded from the mentioned data analysis that, when stressed, the number of mouse usage and keyboard pressing is substantially greater than that of a calm student. Mouse clicks and scroll usage shows a greater hesitation and jitters by the user when accomplishing the proposed task.

In keyboard usage, it is also easy to understand that in a stressed situation, the student uses this device more intensively, with the backspace key having a high frequency of use (Figures 2 and 3). 


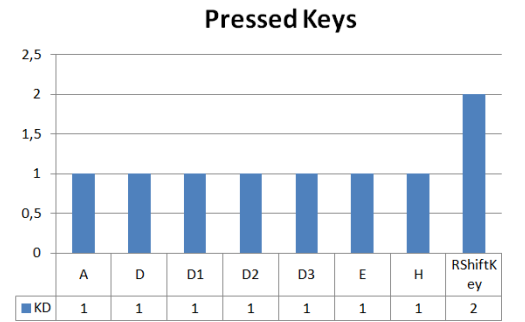

Fig. 2. Pressed Keys without stress.

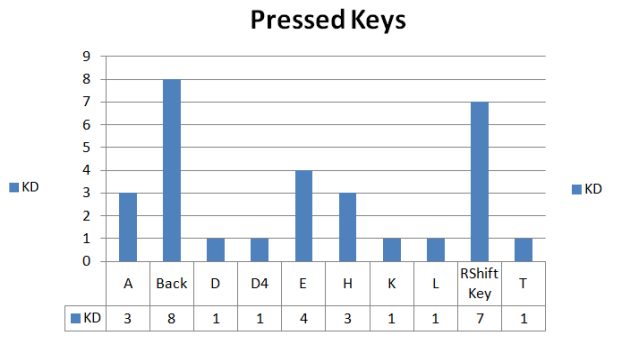

Fig, 3. Pressed Keys in stressed situation.

\section{Conclusions}

It is common sense that stress significantly influences learning capacities, thus learning success. This is particularly relevant when engaging an on-line course, using an E-learning platform, as several research lines indicate. To cope with this factor (together with others), when in an E-learning environment, a Dynamic Student Assessment Module was proposed. This DSAM proposes the use of several usual equipments as sensors, without the user being aware of them. In this paper, particular attention is given to keyboard and mouse, with the development of a log tool to monitor keyboard and mouse usage transparently. A group of programming students was then used to evaluate the hypothesis of stress detection trough mouse and keyboard activity. From the collected data and from posterior analyses it becomes clear that it is highly feasible to detect stress by this method. Significant work is still needed however, and a DSAM as the one proposed in this paper is being developed, to enhance E-learning students' success.

Acknowledgments. This work is funded by National Funds through the FCT Fundação para a Ciência e a Tecnologia (Portuguese Foundation for Science and Technology) within projects PEst-OE/EEI/UI0752/2011 and PTDC/EEI$\mathrm{SII} / 1386 / 2012$. The work of Davide Carneiro is also supported by a doctoral grant by FCT (SFRH/BD/64890/2009).

\section{References}

1. Carneiro, D., Novais, P., Neves, J. (2011): Toward Seamless Environments for Dispute Prevention and Resolution. International Symposium on Ambient Intelligence, Advances in Intelligent and Soft Computing, Springer, Volume 92/2011, DOI: 10.1007/978-3642-19937-0_4. 25-32.

2. Palmer, S.,Cooper,C., and Thomas K (2003): Creating a Balance: Managing Stress British Library London.

3. Rodrigues M., Fdez-Riverola F., Novais P.(2011): Moodle and Affective Computing Knowing Who's on the Other Side, ECEL-2011 - 10th European Conference on Elearning, (University of Brighton, Brighton, UK 10-11 November 2011), ISBN: 978-1908272-22-5, pp 678-685. 
4. C. Fdez-Sampayo; M. Reboiro; D. Glez-Peña; F. Fdez-Riverola (2009): Sistema de seguimiento de actividades en moodle para la evaluación comparativa del ratio de participación alumno/clase. Conferencia Ibero-Americana WWW/Internet 2009: CIAWI 2009. Madrid, Spain. 21 - October.

5. Broekens, J, Jonker, C.M., Meyer, J.J.Ch, (2010): Affective negotiation support systems, Journal of ambient Intelligence and smart Environments, vol2, n. 2

6. Zimmermann, P., Guttormsen, S., Danuser, B., Gomez,P.(2003): Affective ComputingA Rationale For Measuring Mood With Mouse And Keyboard. International Journal Of Occupational Safety And Ergonomics: JOSE Vol. 9, Issue 4, P. 539-551.

7. George A. Tsihrintzis, Maria Virvou, Efthymios Alepis, Ioanna-Ourania Stathopoulou, (2008): Towards Improving Visual-Facial Emotion Recognition through Use of Complementary Keyboard-Stroke Pattern Information. Itng, pp.32-37, Fifth International Conference on Information Technology: New Generation.

8. Castillo, J.C., Rivas-Casado, A., Fernández-Caballero, A., López, M.T., MartínezTomás, R., (2011): A multisensory monitoring and interpretation framework based on the model-view-controller paradigm. In: Proceedings of the 4th International Workshop on the Interplay between Natural and Artificial Computation, vol 1, pp. 441-450.

9. Fernández-Caballero, A., Castillo, J.C., Martínez-Cantos, J., Martínez-Tomás, R., (2010): Optical flow or image subtraction in human detection from infrared camera on mobile robot. Robotics and Autonomous Systems 58 (12), 1273-1281.

10. Dowland, P., \& Furnell, S. (2004): A Long-Term Trial of Keystroke Profiling Using Digraph, Trigraph and Keyword Latencies. In Y. Deswarte, F. Cuppens, S. Jajodia, \& L. Wang (Eds.), Security and Protection in Information Processing Systems (Vol. 147, pp. 275-289). Springer US. doi:10.1007/1-4020-8143-X_18

11. Monrose, F., \& Rubin, A. (1997): Authentication via keystroke dynamics. Proceedings of the 4th ACM conference on Computer and communications security (pp. 48-56). New York, NY, USA: ACM. doi:10.1145/266420.266434

12. ALVES, F., PAGANO, A., \& DA SILVA, I. (2010: A new window on translators' cognitive activity: methodological issues in the combined use of eye tracking, key logging and retrospective protocols. Copenhagen studies in language, (38), 267-291. Retrieved from http://cat.inist.fr/?aModele $=$ afficheN\&cpsidt $=22433271$

13. Freeman, J., \& Ambady, N. (2010): MouseTracker: Software for studying real-time mental processing using a computer mouse-tracking method. Behavior Research Methods, 42(1), 226-241. doi:10.3758/BRM.42.1.226

14. Arroyo, E., Selker, T., \& Wei, W. (2006). Usability tool for analysis of web designs using mouse tracks. CHI '06 Extended Abstracts on Human Factors in Computing Systems (pp. 484-489). New York, NY, USA: ACM. doi:10.1145/1125451.1125557

15. Akamatsu, M., \& MacKenzie, I. S. (2002). Changes in applied force to a touchpad during pointing tasks. International Journal of Industrial Ergonomics, 29(3), 171-182. doi:10.1016/S0169-8141(01)00063-4 